\title{
Ordering a class of unicyclic graphs with respect to Merrifield-Simmons index
}

\author{
Hongzhuan Wang \\ Faculty of Mathematics and Physics, Huaiyin Institute of Technology, Huai'an, Jiangsu 223003, People's Republic of \\ China. E-mail: hongzhuanwang@gmail.com. \\ Accepted 15 December, 2011
}

\begin{abstract}
The Merrifield-Simmons index is one of the most popular topological indices in chemistry and in mathematical properties; there is a correlation between this index and boiling points. The MerrifieldSimmons index of a graph is defined as the total number of its independent sets, including the empty set. This paper proposed an edge grafting theorem operation, which is certain kind of edge moving between two vertices distancing one from the unique cycle. Firstly, we define a new graph which is said to be a cycle-r-regular unicycle graph where each vertex in the unique cycle is with degree $r$. Then we show how the graph of Merrifield-Simmons index changes under the edge grafting operation on the cycle-3-regular unicycle graphs. Finally, we give some applications of these results on ordering the graph of Merrifield-Simmons index among cycle-3-regular unicycle graph. We find that the first three largest values of Merrifield-Simmons index in these graph are $2^{n-3}+2^{n-4}+8,2 \cdot 2^{n-8}-3$ and $3 \cdot 2^{n-7}+8$, respectively.
\end{abstract}

Key words: Independent-vertex set, Merrifield-Simmons index, cycle-3-regular unicyclic graph, external graphs.

\section{INTRODUCTION}

For any given graph $G$, the Merrifield-Simmons index denoted by $\sigma(G)$, is defined as the total number of independent-vertex subsets, including the empty set. The Merrifield-Simmons index was introduced (Prodinger and Tichy, 1982), although it is called Fibonacci number of a graph.

The Merrifield-Simmons index is one of the most popular topological indices in chemistry and its mathematical properties were studied in detail (Prodinger and Tichy, 1982; Xueliang et al., 2005; Pedersen and Vestergard, 2005; Hanyuan et al., 2008; Maolin and Hongbo, 2008a; Hongzhuan and Hongbo, 2006) whereas itsapplicability for quantitative structure-activity relationship (QSAR) and quantitative structure-property relationship (QSPR) was examined to a much lesser extent. For further details on chemical structure (Danesh et al., 2010). Merrifield and Simmons showed the correlation between this index and boiling points (Merrifield and Simmons, 1989). Till now, there have been many papers dealing with Merrifield-Simmons index. Prodinger and Tichy (1982) showed that among all $n$-vertex trees, the star has the maximal Merrifield-Simmons index, while the path has the minimal Merrifield-Simmons index. Alameddine (1998) obtained bounds for the Merrifield-Simmons index of a maximal outerplannar graph. The authors studied the Merrifield-Simmons index of hexagonal chains and catacondensed systems (Gutman, 1993; Lianzhu, 1998), respectively. Xueliang et al. (2005) characterized the tree with the maximal Merrifield-Simmons index among the trees with a given diameter. Pedersen and Vestergard (2005) studied the Merrifield-Simmons index of the unicycle graphs. Hanyuan et al., (2008) studied the upper bound for the Merrifield-Simmons index in $(n, n+1)$ graphs (Hanyuan et al., 2008). Maolin and Hongbo (2008a) determined unique trees with the first and second largest Merrifield-Simmons index, respectively. Hongzhuan and Hongbo (2006) determined the unicycle graphs with the largest and smallest Merrifield-Simmons index. There have been many literatures studying the Merrifield-Simmons index. For further details (Gutman, 1990; Gutman and Kolakovic, 1990; Gutman, 1992).

In order to state and prove our main results, we need 


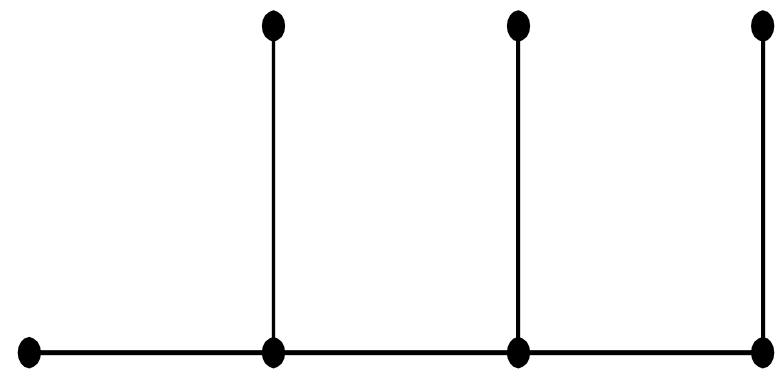

Figure 1. The graph $P_{4}^{2}$ obtained by attaching one pendant edge to each of the vertices of $P_{4}$ respectively except one pendent vertex.

some further notations.

Let $G=(V(G), E(G))$ be a graph with vertex set $V(G)$ and edge set $E(G)$. For any $v \in V(G)$, we denote the neighborhood of $v$ as $N(v)$. Let $[v]=v \cup N(v)$. We use $G-v$ to denote the graph obtained from $G$ by deleting the vertex $v$ and all edges incident with $v$. Let $U_{n}$ denote the set of connected unicycle graphs of order $n$. Let $U_{n}^{r}(l)=\left\{G \in U_{n} \mid d(x)=r, x \in V\left(C_{l}\right)\right\}$, where $r \geq 2$, and $C_{l}$ is the unique cycle in $G$. Any graph in $U_{n}^{r}$ is said to be a cycle-r-regular unicyclic graph. Let $G$ be a connected unicyclic graph and $C_{l}$ be the unique cycle of length $l(3 \leq l \leq n)$ in it. If $n \geq l+1$, then $G$ has at least a vertex with degree one, which is also named a pendent vertex. Let $P_{n}$ be the path on $n$ vertices and its vertices be ordered successively as $x_{1}, x_{2}, \cdots, x_{n}$. By $P_{n}^{k}$ we denote the graph obtained from $P_{n}$ by attaching exactly one pendent edge to each of the vertices $x_{k}, x_{k+1}, \cdots, x_{n}$, respectively. Here, $P_{1}^{1}=P_{2}$ and $P_{2}^{1}=P_{4}$. Let the vertices of $C_{l}$ be ordered successively as $y_{1}, y_{2}, \cdots, y_{l}$. Denote by $C_{n}^{l}\left(t_{1}, t_{2}, \cdots, t_{l}\right)$ the graph obtained from $C_{l}$ by attaching exactly $t_{i}$ pendent edges to the vertex $y_{i}$ for $i=1,2, \cdots, l$, where $t_{i} \geq 0$ and $\sum_{i=1}^{l} t_{i}=n-l$. Here $c_{l}^{l}(0,0, \cdots, 0)=c_{l}$. Let $C_{2 l}^{l}(1,1, \cdots, 1) \infty S_{n-2 l+1}$ be the graph obtained by identifying the center of the star $S_{n-2 l+1}$ and one pendent vertex of $C_{2 l}^{l}(1,1, \cdots, 1)$. Let $C_{2 l}^{l}(1,1, \cdots, 1) \infty\left(S_{k}, S_{n-2 l+1-k}\right)$ be the graph obtained by fusing the centers of two stars $s_{k}, S_{n-21+1-k}$ with two different pendent vertices of the graph $C_{2 l}^{l}(1,1, \cdots, 1)$, respectively. For example, we illustrated $P_{4}^{2}$ in Figure 1, $C_{8}^{4}(1,1,1,1) \infty S_{n-7}, \quad C_{6}^{3}(1,1,1) \infty S_{n-5}$ in Figure 2 and $C_{6}^{3}(1,1,1) \infty\left(S_{n-7}, S_{3}\right)$ in Figure 3, respectively.

Let $T_{n_{1}, n_{2}, \cdots, n_{k}}$ be a tree obtained from a star $S_{k+1}$ by attaching paths of orders $n_{1}, n_{2} \cdots, n_{k}$ to $k$ pendent vertices of $S_{k+1}$. Let $F_{n}$ denote the nth Fibonacci number, then we have $F_{n}+F_{n+1}=F_{n+2}$ with initial conditions $F_{1}=F_{2}=1$.

All graphs considered here are both finite and simple. Other notations and terminology not defined here can be seen in Hongbo (2008).

In this paper, we investigate the Merrifield-Simmons index for graphs in $U_{n}^{3}$, and characterized the $U_{n}^{3}$ with the first, second and third maximal Merrifield-Simmons indices.

\section{SOME LEMMAS}

The following Lemma 1 to 5 can be found in Maolin and Hongbo (2008a), Hongzhuan and Hongbo (2006) and Hongbo (2008).

\section{Lemma 1}

Let $G$ be a graph with $\mathrm{m}$ components $G_{1}, G_{2}, \cdots, G_{m}$. Then $\delta(G)=\prod_{i=1}^{m} \delta\left(G_{i}\right)$.

\section{Lemma 2}

For any graph $G$ with any $v \in V(G)$, we have $\delta(G)=\delta(G-v)+\delta(G-[v])$.

\section{Lemma 3}

For any graph $G$, if $x$ and $y$ are adjacent in $G$, then $\delta(G)=\delta(G-\{x, y\})+\delta(G-[y])+\delta(G-[x])$.

\section{Lemma 4}

For any graph $G$, if $x$ and $y$ are not adjacent in $G$, then 


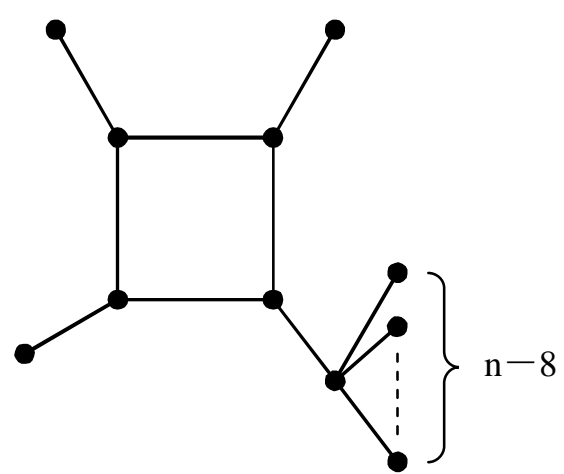

(a)

$$
C_{8}^{4}(1,1,1,1) \infty S_{n-7}
$$

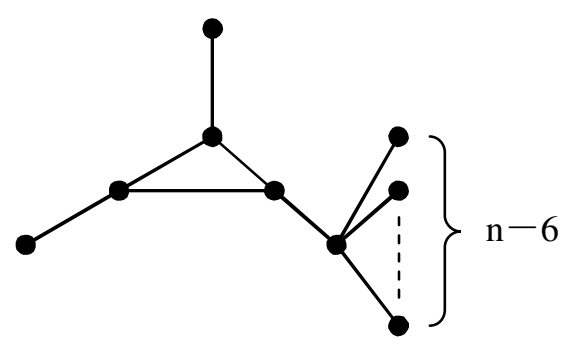

(b)

Figure 2. The graph obtained by identifying the center of star $S_{n-7}\left(\operatorname{resp} . S_{n-5}\right)$ with one pendent vertex of $C_{8}^{4}(1,1,1,1)$ (resp. $\left.C_{6}^{3}(1,1,1)\right)$.



Figure 3. The graph obtained by fusing the center of two stars $S_{3}, S_{n-7}$ with two different pendent vertices $C_{6}^{3}(1,1,1)$.

$\delta(G)=\delta(G-\{x, y\})+\delta(G-x \bigcup[y])+\delta(G-y \bigcup[x])+\delta(G-[x] \bigcup[y])$.

\section{Lemma 5}

Let $T$ be a tree, then $F_{n+2} \leq \delta(T) \leq 2^{n-1}+1$ and $\delta(T)=F_{n+2}$ if and only if $T \cong P_{n}$, and $\delta(T)=2^{n-1}+1$ if and only if $T \cong S_{n}$.

\section{Remark 1}

As in many previous works, people discuss the properties of the Merrifield-Simmons index for molecular graphs representing hydrocarbons with emphasis on molecular trees, unicycle graphs and bicycle graphs. In this work, we consider a new graph cycle-3-regular unicycle graph.

\section{THE MAXIMAL VALUE OF MERRIFIELD-SIMMONS INDEX IN $U_{n}^{3}$}

In this section, we study how Merrifield-Simmons index changes under certain graph operations (in Lemma 6 and Lemma 7) on cycle-3-regular graphs. This result will be used throughout the paper.

\section{Lemma 6}

Suppose $s \geq 1$ is an integer, let $u$ be a pedant vertex of a connected graph $G_{0}$ with at least two vertices. Let $G$ be the graph obtained by identifying $u$ and a pendent vertex of a star $S_{s+2}$, Let $G^{\prime}$ be the graph obtained by identifying $u$ and the center of a star $S_{s+2}$, Then $\delta\left(G^{\prime}\right)>\delta(G)$.

\section{Proof}

By Lemma 2, we have: 

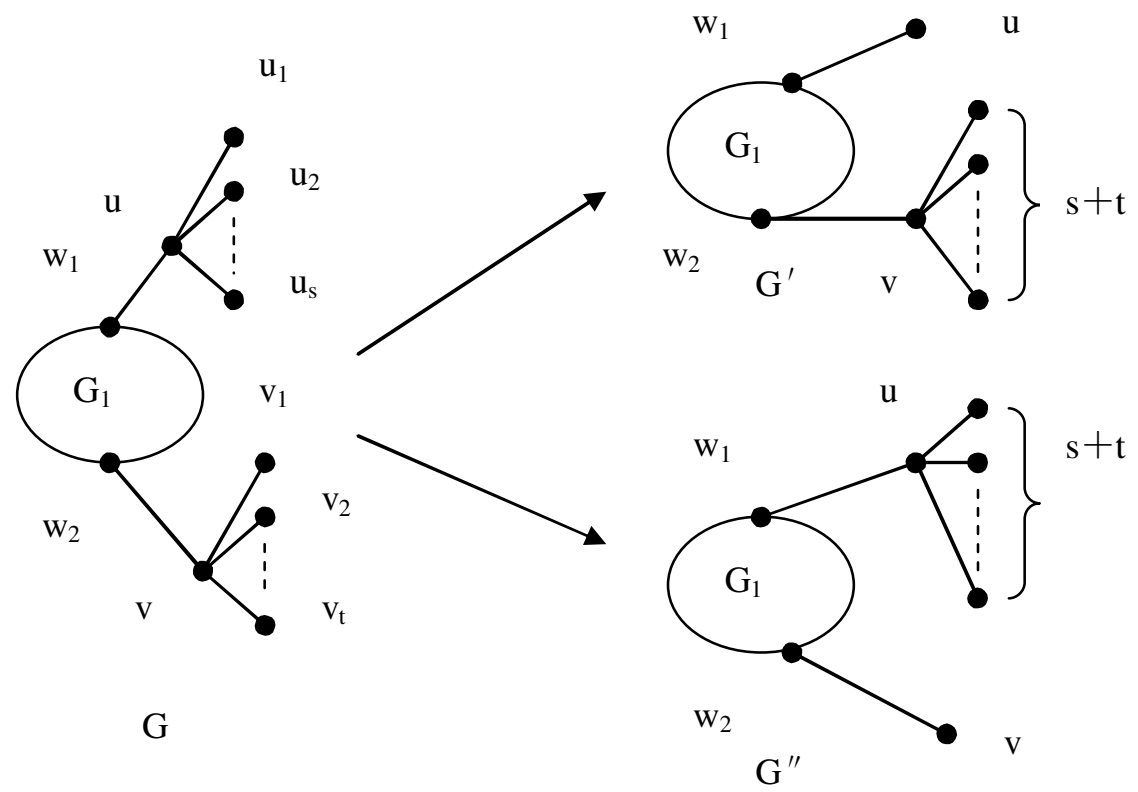

Figure 4. Operation that increases the Merrifield-Simmons index of cycle-3-regular graphs.

$\delta(G)=\delta(G-u)+\delta(G-[u])=\left(2^{s}+1\right) \delta\left(G_{0}\right)+2^{s} \delta\left(G_{0}-w\right)$.

While, $\quad \delta\left(G^{\prime}\right)=\delta(G-u)+\delta(G-[u])=2^{s+1} \delta\left(G_{0}\right)+\delta\left(G_{0}-w\right)$. Where $w$ be the neighborhood of $u$ in $G_{0}$. Hence, $\delta\left(G^{\prime}\right)-\delta(G)=\left(2^{s}-1\right)\left[\delta\left(G_{0}\right)-\delta\left(G_{0}-w\right)\right]>0$. Therefore, $\delta\left(G^{\prime}\right)>\delta(G)$.

This finished the proof of Lemma 6.

\section{Lemma 7}

Let $u$ and $v$ be pendent vertices of a connected graph $G_{1}$ with at least two vertices. Let $u_{1}, u_{2}, \cdots, u_{s}$ be the leaves adjacent to $u$ in $G$, Let $v_{1}, v_{2}, \cdots, v_{t}$ be the leaves adjacent to $v$ in $G$, We suppose that $G^{\prime}=G-\left\{u u_{1}, u u_{2}, \cdots, u u_{s}\right\}+\left\{v u_{1}, v u_{2}, \cdots, v u_{S}\right\} \quad$ and $G^{\prime \prime}=G-\left\{v v_{1}, v v_{2}, \cdots, v v_{t}\right\}+\left\{u v_{1}, u v_{2}, \cdots, u v_{t}\right\} . \quad$ (Figure 4), then either $\delta\left(G^{\prime}\right)>\delta(G)$ or $\delta\left(G^{\prime \prime}\right)>\delta(G)$.

\section{Proof}

It is easy to see that $G_{1}=G-\left\{u, v, u_{1}, u_{2}, \cdots, u_{s}, v_{1}, v_{2}, \cdots v_{t}\right\}$.
Because $u, v$ are not adjacent in $G, G^{\prime}$ and $G^{\prime \prime}$ by the definition of Merrifield-Simmons index and by Lemma 4, we have:

$\delta(G)=\delta(G-\{u, v\})+\delta(G-u U[v])+\delta(G-v(u])+\delta(G-[u][v])$

$=2^{s+t} \delta\left(G_{1}\right)+2^{s} \delta\left(G_{1}-w_{2}\right)+2^{t} \delta\left(G_{1}-w_{1}\right)+\delta\left(G_{1}-\left\{w_{1}, w_{2}\right\}\right)$

and

$\delta(G)=\delta(G-\{u, v\})+\delta(G-u \bigcup[v])+\delta(G-v \bigcup[u])+\delta(G-[u] \bigcup[v])$

$=2^{s+t} \delta\left(G_{1}\right)+\delta\left(G_{1}-w_{2}\right)+2^{s+t} \delta\left(G_{1}-w_{1}\right)+\delta\left(G_{1}-\left\{w_{1}, w_{2}\right\}\right)$

respectively, where $w_{1}, w_{2}$ denote the neighborhood of $u$ and $v$ in $G_{1}$, For the difference contributions for $\delta(G)$ and $\delta\left(G^{\prime}\right)$, we get $\Delta_{1}=\delta(G)-\delta(G)=\left(2^{s}-1\right)\left[2^{t} \delta\left(G_{1}-w_{1}\right)-\delta\left(G_{1}-w_{2}\right)\right]$.

Similarly, there is $\delta\left(G^{\prime \prime}\right)=\delta\left(G^{\prime \prime}-\{u, v\}\right)+\delta\left(G^{\prime \prime}-u \bigcup[v]\right)+\delta\left(G^{\prime \prime}-v \bigcup[u]\right)+\delta\left(G^{\prime \prime}-[u] \bigcup[v]\right)$. $=2^{s+t} \delta\left(G_{1}\right)+2^{s+t} \delta\left(G_{1}-w_{2}\right)+\delta\left(G_{1}-w_{1}\right)+\delta\left(G_{1}-\left\{w_{1}, w_{2}\right\}\right)$

For the difference contributions for $\delta(G)$ and $\delta\left(G^{\prime \prime}\right)$, we get 
$\Delta_{2}=\delta\left(G^{\prime \prime}\right)-\delta(G)=\left(2^{t}-1\right)\left[2^{s} \delta\left(G_{1}-w_{2}\right)-\delta\left(G_{1}-w_{1}\right)\right]$.

If $\delta\left(G^{\prime}\right)<\delta(G), \quad$ that is, $\quad \Delta_{1}<0$, then

$\delta\left(G_{1}-w_{2}\right) \geq 2^{t} \delta\left(G_{1}-w_{1}\right) . \quad$ There

is

$\Delta_{2}=\left(2^{t}-1\right)\left[2^{s} \delta\left(G_{1}-w_{2}\right)-\delta\left(G_{1}-w_{i}\right)\right] \geq\left(2^{t}-1\right)\left(2^{s+t}-1\right) \delta\left(G_{1}-w_{1}\right)>0$.

This finishes the proof of Lemma 7.

It follows that the value of Merrifield-Simmons index is strictly increasing by this operation. This in turn provides a way of comparing the Merrifield-Simmons index.

By the proof of Lemmas 6 and 7, we can verify that the external graph with maximal Merrifield-Simmons index must be the graph such that all the pendent edges not on the cycle are attached to the same vertex out of the cycle. Therefore, we obtain the following Lemma 8.

\section{Remark 2}

The edge grafting operations on the graphs were often considered and used in the study of the invarant structure, for example, Kexiang and Kinkar (2011) characterized the extremal graphs with the lower or upper bounds on the Harary index. Hongbo and Shenggui (2011) characterized the graphs with the maximum and minimum Merrifield-Simmons index, respectively among the graphs given cut vertices. This paper also uses this transformation to obtain optimal solution for comparison of the Merrifield-Simmons index in cycle-3-regular graph. Other methods used in studying the chemical structure can be seen in Mollaamin et al. (2011) and Zhang et al. (2011).

\section{Lemma 8}

If $G \in U_{n}^{3}(l)$, then $\delta(G) \leq \delta\left(C_{2 l}^{l}(1,1, \cdots, 1) \infty S_{n-2 l+1}\right)$ with the equality if and only if $G \cong C_{2 l}^{l}(1,1, \cdots, 1) \infty S_{n-2 l+1}$.

\section{Lemma 9}

Let $l \geq 5$ and $n \geq 2 l$ be integer, then

$$
\delta\left(C_{2 l}^{l}(1,1, \cdots, 1) \infty S_{n-2 l+1}\right)<\delta\left(C_{2(l-1)}^{l-1}(1,1, \cdots, 1) \infty S_{n-2 l+3}\right) .
$$

\section{Proof}

By Lemma 1 and by the definition of Merrifield-Simmons index, we have
$\delta\left(C_{2 l}^{l}(1,1, \cdots, 1) \infty S_{n-2 l+1}\right)=2^{n-2 l} \delta\left(C_{2^{l}}^{l}(0,1, \cdots, 1)\right)+\delta\left(P_{l-1}^{1}\right)$

$\delta\left(C_{2(l-1)}^{l-1}(1,1, \cdots, 1) \propto S_{n-2 l+3}\right)=2^{n-2 l+2} \delta\left(C_{2(l-1)}^{l-1}(0,1, \cdots, 1)\right)+\delta\left(P_{l-2}^{1}\right)$

In the following, we first simplify the first part of Equations 1 and 2. Let

$$
F_{0}=C_{2 l}^{l}(0,1, \cdots, 1), \quad F_{1}=C_{2(l-1)}^{l-1}(0,1, \cdots, 1)
$$

At the same time, let $u, u^{\prime} \in C_{l}$ and $d(u)=d\left(u^{\prime}\right)=2$. By Lemma 2, we have

$\delta\left(C_{2 l}^{l}(0,1, \cdots, 1)\right)=\delta\left(F_{0}\right)=\delta\left(F_{0}-u\right)+\delta\left(F_{0}-[u]\right)=\delta\left(P_{l-1}^{1}\right)+4 \delta\left(P_{l-3}^{1}\right)$

and

$\delta\left(C_{2(l-1)}^{l-1}(0,1, \cdots, 1)\right)=\delta\left(F_{1}\right)=\delta\left(F_{1}-u^{\prime}\right)+\delta\left(F_{0}-\left[u^{\prime}\right]\right)=\delta\left(P_{l-2}^{1}\right)+4 \delta\left(P_{l-4}^{1}\right)$, respectively.

It can be easily seen that

$\delta\left(P_{l-1}^{1}\right)=2 \delta\left(P_{l-2}^{1}\right)+2 \delta\left(P_{l-3}^{1}\right)$.

Moreover, we have the following

$4 \delta\left(P_{l-3}^{1}\right)<\delta\left(P_{l-1}^{1}\right)<4 \delta\left(P_{l-2}^{1}\right)$.

Similarly, we have $4 \delta\left(P_{l-5}^{1}\right)<\delta\left(P_{l-3}^{1}\right)<4 \delta\left(P_{l-4}^{1}\right)$.

Hence,

(2) $-(1)$

$=2^{n-2 l}\left[\left(4 \delta\left(P_{l-2}^{1}\right)-\delta\left(P_{l-1}^{1}\right)\right)+4\left(4 \delta\left(P_{l-4}^{1}\right)-\delta\left(P_{l-3}^{1}\right)\right)-\left[\delta\left(P_{l-1}^{1}\right)-\delta\left(P_{l-2}^{1}\right)\right]\right.$

$=2^{\mathrm{n}-2 \mathrm{l}}\left[2 \delta\left(P_{l-3}^{1}\right)+4 \delta\left(P_{l-4}^{1}\right)+16 \delta\left(P_{l-4}^{1}\right)-4 \delta\left(P_{l-3}^{1}\right)\right]-\left[\delta\left(P_{l-1}^{1}\right)-\delta\left(P_{l-2}^{1}\right)\right]$

$=2^{\mathrm{n}-21}\left[20 \delta\left(P_{l-4}^{1}\right)-2 \delta\left(P_{l-3}^{1}\right)\right]-\left[\delta\left(P_{l-1}^{1}\right)-\delta\left(P_{l-2}^{1}\right)\right]$

$=2^{\mathrm{n}-2 \mathrm{l}}\left[16 \delta\left(P_{l-4}^{1}\right)-4 \delta\left(P_{l-5}^{1}\right)\right]-\left[\delta\left(P_{l-1}^{1}\right)-\delta\left(P_{l-2}^{1}\right)\right]$

$>2^{n-2 l} \cdot 14 \delta\left(P_{l-4}^{1}\right)-\left(4 \delta\left(P_{l-3}^{1}\right)+2 \delta\left(P_{l-4}^{1}\right)\right)$

$>2^{n-2 l} \cdot 14 \delta\left(P_{l-4}^{1}\right)-18 \delta\left(P_{l-4}^{1}\right)$

$=2^{n-2 l-1} \cdot 28 \delta\left(P_{l-4}^{1}\right)-18 \delta\left(P_{l-4}^{1}\right)$

$>\left(2^{n-2 l-1}-1\right) \cdot 18 \delta\left(P_{l-4}^{1}\right)>0$. 


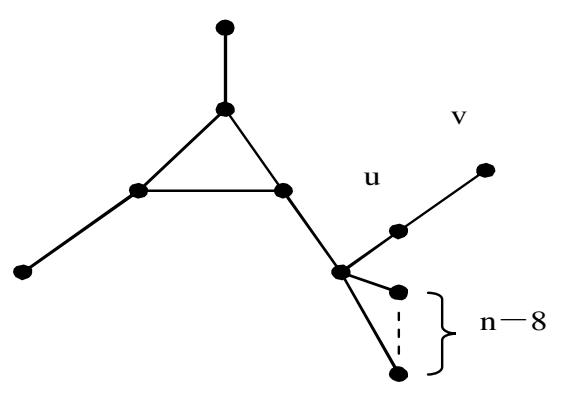

$\mathrm{T}_{1}$

Figure 5. The graphs in Theorem 11.

Therefore,

$\delta\left(C_{2 l}^{l}(1,1, \cdots, 1) \infty S_{n-2 l+1}\right)<\delta\left(C_{2(l-1)}^{l-1}(1,1, \cdots, 1) \infty S_{n-2 l+3}\right)$.

For all $l \geq 5$. This completes the proof.

Next, we will give applications of the above results (about the effect of edge grafting operation) to the comparison of the Merrifield-Simmons index of cycle-3regular unicycle graphs.

\section{Theorem 10}

Let $G$ be a graph of order $n \geq 10$ in $U_{n}^{3}$. Then $\delta(G) \leq 2^{n-3}+2^{n-4}+8$, with equality if and only if $G \cong C_{6}^{3}(1,1,1) \infty S_{n-5}$.

\section{Proof}

For $l \geq 5$ and $n \geq 2 l$, by direct verification and Lemma 9 , it follows that the value of Merrifield-Simmons index in cycle-3-regular graph is decreasing for $l \geq 5$ and $n \geq 2 l$. Therefore, for any graph $G$ in $U_{n}^{3}$, there is $\delta(G) \leq \delta\left(C_{8}^{4}(1,1,1,1) \infty S_{n-7}\right)$.

Finally we need to compare the Merrifield-Simmons indices of $C_{8}^{4}(1,1,1,1) \infty S_{n-7}$ and $C_{6}^{3}(1,1,1) \infty S_{n-5}$. Let $G=c_{8}^{4}(1,1,1,1) \infty S_{n-7}$ and $G^{\prime}=C_{6}^{3}(1,1,1) \infty S_{n-5}$.

By direct calculation, we obtain $\delta(G)=2^{n-8} \cdot 22+2^{n-6} \cdot 3+22 \quad$ and $\delta\left(G^{\prime}\right)=2^{n-3}+2^{n-4}+8$, respectively.

Since $\delta\left(G^{\prime}\right)-\delta(G)=2^{n-7} \cdot 7-14>0(n \geq 10)$, we complete the proof.

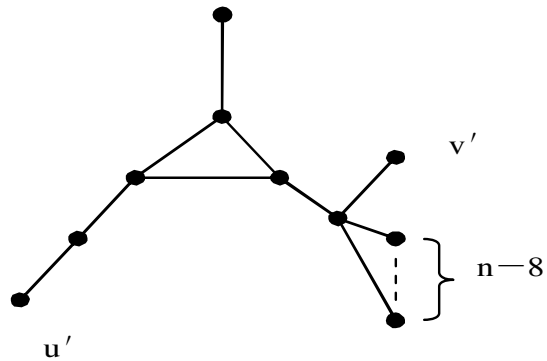

$\mathrm{T}_{2}$

THE SECOND MAXIMAL VALUE OF MERRIFIELDSIMMONS INDEX IN $U_{n}^{3}$

In this section, we characterize the cycle-3-regular graph with the second maximal Merrifield-Simmons index. Let $T_{1}=C_{6}^{3}(1,1,1) \infty T(1,1, \cdots, 2) \quad$ and $T_{2}=C_{6}^{3}(1,1,1) \infty\left(S_{n-6}, S_{2}\right)$ (Figure 5).

\section{Theorem 11}

Let $n \geq 10$ be an integer, $G \in\left\{U_{n}^{3}-C_{6}^{3}(1,1,1) \infty S_{n-5}\right\}$. Then $\quad \delta(G) \leq 2^{n-8} \cdot 2-3$ with equality if and only if $G \cong T_{2}$.

\section{Proof}

Suppose that $G$ has the second maximal MerrifieldSimmons index in $U_{n}^{3}$. By Lemmas 6 and 7 , we conclude that $G$ must be of the form $T_{1}$ or $T_{2}$. In the following, we will get the second maximal Merrifield-Simmons index in $U_{n}^{3}$. By direct calculations, we get:

$$
\begin{aligned}
& \delta\left(T_{1}\right)=\delta\left(T_{1}-\{u, v\}\right)+\delta\left(T_{1}-[v]\right)+\delta\left(T_{1}-[u]\right) \\
& =\delta\left(C_{6}^{3}(1,1,1) \propto S_{n-7}\right)+2^{n-8} \delta\left(C_{5}^{3}(1,1,0)\right)+\delta\left(C_{6}^{3}(1,1,1) \propto S_{n-7}\right) \\
& =2 \delta\left(C_{6}^{3}(1,1,1) \propto S_{n-7}\right)+2^{n-8} \delta\left(C_{5}^{3}(1,1,0)\right)
\end{aligned}
$$

and

$\left.\left.\delta T_{2}\right)=\delta T_{2}-\left\{u^{\prime}, v^{\prime}\right\}\right)+\delta T_{2}-u^{\prime} \bigcup\left[v^{\prime}\right]+\delta T_{2}-v^{\prime} \bigcup\left[u^{\prime}\right]+\delta T_{2}-\left[v^{\prime}\right] \bigcup\left[u^{\prime}\right]$ 


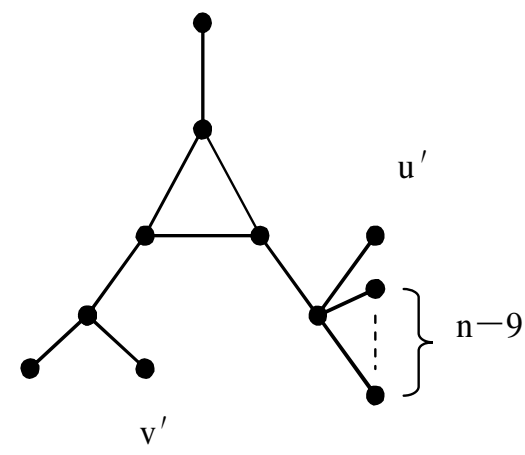

$\mathrm{T}_{3}$

Figure 6. The graphs in Theorem 12.

$\left.\left.\left.=\delta C_{6}^{3}(1,1,1) \propto \Phi_{n-7}\right)+2^{n-8} \delta\left(C_{5}^{3}(1,1,0)\right)+\delta C_{5}^{3}(1,1,1) \propto \Phi_{n-7}\right)+2^{n-8} \delta C_{4}^{3}(1,1,0)\right)$, respectively. Where $u v$ be pendent edge of $T_{1}$ and $u^{\prime}, v^{\prime}$ be different pendent vertices of $T_{2}$.

Hence,

$\delta\left(T_{2}\right)-\delta\left(T_{1}\right)=\delta\left(C_{5}^{3}(1,1,0) \propto S_{n-7}\right)+2^{n-8} \delta\left(C_{4}^{3}(1,0,0)\right)-\delta\left(C_{6}^{3}(1,1,1) \propto S_{n-7}\right)$

$\left.\left.=\delta\left(C_{5}^{3}(1,1,0) \infty S_{n-7}\right)+2^{n-8} \delta C_{4}^{3}(1,0,0)\right)-\left[\delta C_{5}^{3}(1,1,0) \infty S_{n-7}\right)+\delta(T(1,1, \cdots 1,3))\right]$

$=2^{n-8} \delta\left(C_{4}^{3}(1,0,0)\right)-\delta\left(T(1,1, \cdots ; 1,3)=2^{n-8} \delta\left(C_{4}^{3}(1,0,0)\right)-\left[2^{n-8} \cdot \delta\left(P_{3}\right)+\delta\left(P_{2}\right)\right]\right.$

$=2^{n-8}\left(2 \cdot \delta\left(P_{2}\right)+1\right)-2^{n-8} \cdot \delta\left(P_{3}\right)-\delta\left(P_{2}\right)=2^{n-8} \cdot 2-3>0 \quad(n \geq 10)$.

This completes the proof of Theorem 11.

\section{THE THIRD MAXIMAL VALUE OF MERRIFIELD- SIMMONS INDEX IN $U_{n}^{3}$}

In this section, we characterized the cycle-3-regular graph with the third maximal Merrifield-Simmons index. Let $\quad T_{3}=C_{6}^{3}(1,1,1) \infty\left(S_{n-7}, S_{n-3}\right)$ $, T_{4}=C_{6}^{3}(1,1,1) \infty\left(S_{2}, T(1,1, \cdots 1,2)\right),($ Figure 6)

\section{Theorem 12}

Among all graphs in $U_{n}^{3}$ with $n \geq 10$, the graph

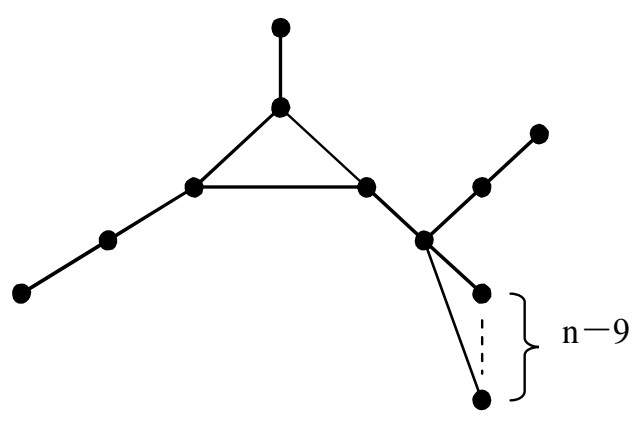

$\mathrm{T}_{4}$

Table 1. Some results on Hosoya index.

\begin{tabular}{lll}
\hline Hosoya index & Largest & Smallest \\
\hline Trees of order $\mathrm{n}$ & $P_{n}$ & $S_{n}$ \\
Unicycle graphs of order $\mathrm{n}$ & $C_{n}$ & $S_{n}+e$ \\
Bicycle graphs of order $\mathrm{n}$ & $H_{0}$ or $K_{2,3}$ & $F_{0}$ \\
\hline
\end{tabular}

Table 2. Some results on Merrifield-Simmons index.

\begin{tabular}{lll}
\hline Merrifield-Simmons & Largest & Smallest \\
\hline Trees of order $\mathrm{n}$ & $S_{n}$ & $P_{n}$ \\
Unicycle graphs of order $\mathrm{n}$ & $S_{n}+e$ or $C_{4}$ & $C_{n}$ \\
Bicycle graphs of order $\mathrm{n}$ & $F_{0}$ & $H_{0}$ or $K_{2,3}$ \\
\hline
\end{tabular}

where $F_{0}$ is obtained from $S_{n}$ by adding two adjacent edges. $H_{0}$ is the graph connecting two 3-cycle by a path of length $n-5 . K_{2,3}$ is the bipartite graph (Hanyuan et al., 2006; Pedersen and Vestergaard, 2005; Gutman, 1990; Hanyuan, 2008a; Prodinger and Tichy, 1982; Hanyuan, 2008b).

$C_{6}^{3}(1,1,1) \infty T(1,1, \cdots, 1,2)$, (that is, $\left.T_{1}\right)$ has the third maximal Merrifield-Simmons index, and $\delta\left(T_{1}\right)=3 \cdot 2^{n-7}+8$. 


\section{Proof}

We denote by $G$ the graph in $U_{n}^{3}$ with the third largest Merrifield-Simmons index, by Lemma 6 and 7, $G$ must be the form of $T_{1}, T_{3}$ or $T_{4}$ (Figure 6). By Lemma 7, we obviously have $\delta\left(T_{4}\right)<\delta\left(T_{1}\right)$. Next, we will show that $\delta\left(T_{1}\right)>\delta\left(T_{3}\right)$. For $u, v$ are adjacent in $T_{1}$, by Lemma 3 , we have:

$$
\begin{aligned}
& \delta\left(T_{1}\right)=\delta\left(T_{1}-\{u, v\}\right)+\delta\left(T_{1}-[v]\right)+\delta\left(T_{1}-[u]\right) \\
& =2 \delta\left(C_{6}^{3}(1,1,1) \infty S_{n-7}\right)+2^{n-8} \delta\left(C_{5}^{3}(1,1,0)\right) \\
& =2\left[\delta\left(C_{6}^{3}(1,1,1) \infty S_{n-8}\right)+2^{n-9} \delta\left(C_{5}^{3}(1,1,0)\right)\right]+2^{n-8} \delta\left(C_{5}^{3}(1,1,0)\right) \\
& =2 \delta\left(C_{6}^{3}(1,1,1) \infty S_{n-8}\right)+2^{n-7} \delta\left(C_{5}^{3}(1,1,0)\right)
\end{aligned}
$$

If $u^{\prime}, v^{\prime}$ are not adjacent in $T_{3}$, then by Lemma 4, we have

$$
\begin{aligned}
& \delta\left(T_{3}\right)=\delta\left(T_{3}-\left\{u^{\prime}, v^{\prime}\right\}\right)+\delta\left(T_{3}-u^{\prime} \bigcup\left[v^{\prime}\right]\right)+\delta\left(T_{3}-v^{\prime} \bigcup\left[u^{\prime}\right]\right)+\delta\left(T_{3}-\left[v^{\prime}\right] \bigcup\left[u^{\prime}\right]\right) \\
& =\delta\left(C_{6}^{3}(1,1,1) \infty S_{n-8}\right)+\delta\left(C_{5}^{3}(1,1,0) \infty S_{n-8}\right)+2 \delta\left(C_{5}^{3}(1,1,0) \infty S_{n-8}\right) \\
& +2 \cdot 2^{n-9} \delta\left(C_{4}^{3}(1,1,0)\right)+2^{n-9}\left[\delta\left(C_{5}^{3}(1,1,0)\right)+\delta\left(C_{4}^{3}(1,1,0)\right)\right] \\
& =\delta\left(C_{6}^{3}(1,1,1) \infty S_{n-8}\right)+3 \delta\left(C_{5}^{3}(1,1,0) \infty S_{n-8}\right) \\
& +3 \cdot 2^{n-9} \delta\left(C_{4}^{3}(1,1,0)\right)+2^{n-9} \delta\left(C_{5}^{3}(1,1,0)\right) .
\end{aligned}
$$

For the difference of contribution for $\delta\left(T_{1}\right)$ and $\delta\left(T_{3}\right)$, we get:

$$
\begin{aligned}
& \Delta_{3}=\delta\left(T_{1}\right)-\delta\left(T_{3}\right)=\left[\delta\left(C_{6}^{3}(1,1,1) \infty S_{n-8}\right)-3 \delta\left(C_{5}^{3}(1,1,0) \infty S_{n-8}\right)\right] \\
& +\left[2^{n-9} \cdot 3 \delta\left(C_{5}^{3}(1,1,0)\right)-3 \cdot 2^{n-9} \delta\left(C_{4}^{3}(1,1,0)\right)\right] \\
& =\left[\delta\left(T(1,1, \cdots, 1,3)-2 \delta\left(C_{5}^{3}(1,1,0) \infty S_{n-8}\right)\right]\right. \\
& +2^{n-9} \cdot 3\left[\delta\left(C_{5}^{3}(1,1,0)\right)-\delta\left(C_{4}^{3}(1,1,0)\right)\right] \\
& =-\delta\left(T(1,1, \cdots, 1,3)-4 \delta\left(S_{n-8}\right)+2^{n-9} \cdot 3 \delta\left(P_{3}\right) .\right.
\end{aligned}
$$

By Lemma 5, we have

$$
\begin{aligned}
& \Delta_{3}=-\left[\delta\left(P_{3}\right) \cdot 2^{n-9}+\delta\left(P_{2}\right)\right]-4\left(2^{n-9}+1\right)+2^{n-9} \cdot 3 F_{5} \\
& =-F_{5} \cdot 2^{n-9}-F_{4}-4\left(2^{n-9}+1\right)+2^{n-9} \cdot 3 F_{5} \\
& =2^{n-8} \cdot F_{5}-F_{4}-2^{n-7}-4=2^{n-8} \cdot 3-7>0 \quad(n \geq 10) .
\end{aligned}
$$

Which gives $\delta\left(T_{1}\right)>\delta\left(T_{3}\right)$. This proves the present theorem. When $6 \leq n \leq 9$, all results in this paper still hold.

\section{Remark 3}

It is surprising that the graphs with the largest MerrifieldSimmons index among the cycle-3-regular unicycle graph are the same as those with the smallest energy (Maolin and Hongbo, 2008b).

Finally, we survey some results on the extremal graphs for the Hosoya index and Merrifield-Simmons index in trees, unicycle graphs and bicycle graphs (Tables 1 and 2). From the survey, we posed a problem.

\section{Problem}

Within any given set of nontricial connected graphs, is the graph with maximal Merrifield-Simmons index just one with minimal Hosoya index?

Obviously, the answer to this problem is positive for the sets tree, unicycle graphs and bicycle graphs, respectively. But for other general given sets of graphs (such as cycle-3-regular graph), the answer to it is still unknown, maybe it will be an interesting topic for the further research in the future.

\section{CONCLUSIONS}

In this paper, we considered a new class of unicycle graph cycle-3-regular unicycle graph and the famous Merrifield-Simmons index. We introduced an efficient graph operation method. Using this new method, we were able to provide a simplified proof of the above mentioned orders of the cycle-3-regular unicycle graphs. It seems that the graph with the first three largest Merrifield-Simmons index in cycle-r-regular unicycle graphs $(r \geq 4)$ should have a similar structure as the one in cycle-3-regular unicycle graphs. So, the same reasoning should work well for discussing the first three largest Merrifield-Simmons index for graphs in cycle-rregular unicycle graphs $(r \geq 4)$.

\section{REFERENCES}

Alameddine AF (1998). Bounds on the Fibonacci number of a maximal 
outerplanar graph. Fib. Quart, 36: 206-210.

Danesh M, Shirgahi H, Danesh N (2010). Improving multiplication and reminder using implementation based on word and index. Int. J. Phys. Sci., 5(8): 1290-1295.

Gutman I (1993). Extremal hexagonal chains. J. Math. Chem., 12: 197210.

Gutman I (1990). Fragmentation formulas for the number of kekule structure, Hosoya and Merrifield-Simmons indices and related graph invariants. Coll. Sci. Pap. Fac. Sci. Kragnjevac., 11: 11-18.

Gutman I, Kolakovic N (1990). Hosoya index of the second of molecules containing a polyacene fragment. Bril. Acad. Serbe Sci. Arts (Cl. Math. Natu)., 102: 39-46.

Gutman I (1992). Independent vertex sets in some compound graphs. Publ. Inst. Math. (Beograd), 52: 5-9.

Hanyuan D, Chen X, Zhang J (2008). The Merrifield-Simmons index in $(n, n+1)$-graphs. J. Math. Chem., 43: 75-90.

Hanyuan D (2008a). The smallest Hosoya index in (n, $n+1)$-graphs. J. Math. Chem., 1(43): 119-133.

Hanyuan D (2008b). The largest Hosoya index in $(n, n+1)$-graphs. Math. Comput. Appl., 10(56): 2499-2506.

Hongbo H (2008). Minimizing a class of unicyclic graphs by means of Hosoya index. Math. Comput. Model., 48: 940-948.

Hongbo H, Shenggui Z (2011). Graphs with given number of cut vertices and extremal Merrifield-Simmons. Discrete Appl. Math., 159: 971-980.

Hongzhuan W, Hongbo H (2006). Unicycle graphs with extremal Merrifield-Simmons index. J. Math. Chem., 43: 202-209.

Kexiang X, Kinkar CH (2011). On Harary index of graphs. Discrete Appl. Math., 159: 1631-1640.

Lianzhu ZZ (1998). The proof of Gutman's conjectures concerning extremal hexagonal chains. J. Sys. Sci., 18: 460-465.

Maolin H, Hongbo H (2008a). The first and second largest MerrifieldSimmons indices of trees with prescribed pendent vertices. J. Math. Chem., 43: 727-736.
Maolin W, Hongbo H (2008b). Minimal energy on class of graphs. J. Math. Chem., 4 (43): 1389-1402.

Merrifield RE, Simmons HE (1989). Topological Methods in Chemistry. Wiley, New York, 30: 130-145.

Mollaamin F, Gharibe S, Monajjemi M (2011). Synthesis of Various nano and micro ZnSe morphologies by using hydrothermal method. Int. J. Phys. Sci., 6 (6): 1496-1500.

Pedersen AS, Vestergaard PD (2005). The number of independent sets in unicyclic graphs, Discrete Appl. Math., 152: 246-256.

Prodinger H, Tichy RF (1982). Fibonacci numbers of graphs. Fib. Quart., 20 (1): 16-21.

Xueliang L, Zhao H, Gutman I (2005). On the Merrifield-Simmons index of trees. MATCH Commun. Math. Comput. Chem., 54: 389-402.

Zhang S, Xie M, Zhang Y (2011). A novel method for object detection based on graph theory. Int. J. Phys. Sci., 6(29): 6735-6740. 